\title{
NOVOS CONSTITUINTES QUÍMICOS DAS CASCAS DO CAULE DE Tabebuia heptaphylla
}

Fernanda Rodrigues Garcez*, Walmir Silva Garcez, Talal Suleiman Mahmoud e Patrícia de Oliveira Figueiredo Departamento de Química, Universidade Federal de Mato Grosso do Sul, CP 649, 79070-900 Campo Grande - MS, Brasil Ubirazilda Maria Resende

Departamento de Biologia, Universidade Federal de Mato Grosso do Sul, 79070-900 Campo Grande - MS, Brasil

Recebido em 20/10/06; aceito em 10/4/07; publicado na web em 25/9/07

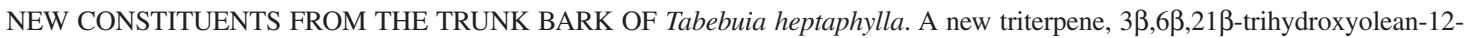
ene and a new iridoid, $8 \alpha$-methyl-8$\beta$-hydroxy- $6 \beta$-(3',4'-dimethoxy)benzoyloxy-1 $\alpha, 3 \alpha$-dimethoxy-octahydro-cyclopenta[c]pyran were isolated from the trunk bark of a specimen of Tabebuia heptaphylla (Bignoniaceae) collected in the "Pantanal" of Mato Grosso do Sul, Brazil. Twelve known compounds were also obtained in this work, comprising four iridoids, 6-O-p-hydroxybenzoylajugol, 6- $O$ - $p$-methoxybenzoylajugol, 6- $O-3$ ",4"'-dimethoxybenzoylajugol, $8 \alpha$-methyl-8 $\beta$-hydroxy-6 $\beta$-(4'-hydroxy)benzoyloxy- $1 \alpha, 3 \alpha-$ dimethoxy-octahydro-cyclopenta[c]pyran, a cyclopentene dialdehyde, 2-formyl-5-(3',4'-dimethoxybenzoyloxy)-3-methyl-2cyclopentene-1-acetaldehyde, a phenylethanoid glycoside, verbascoside and three benzoic acid derivatives, $p$-hydroxybenzoic, $p$ methoxybenzoic and 3,4-dimethoxybenzoic acids, in addition to squalene, sitostenone and sitosterol. The antioxidant properties of the isolated compounds were also evaluated in this work.
\end{abstract}

Keywords: Tabebuia heptaphylla; triterpenes; iridoids.

\section{INTRODUÇÃO}

Bignoniaceae, família que engloba aproximadamente 120 gêneros e 800 espécies, possui representantes nas regiões tropicais e subtropicais de todo o mundo, especialmente na América do Sul e África ${ }^{1}$. As espécies desta família pertencentes ao gênero Tabebuia, encontradas nas Américas do Sul e Central, são conhecidas popularmente como "ipês" e apresentam uma grande diversidade de constituintes químicos, notadamente naftoquinonas e seus derivados, além de iridóides e antraquinonas ${ }^{2-4}$.

Tabebuia heptaphylla (Vell.) Toledo tem porte arbóreo, atingindo de 10 a $20 \mathrm{~m}$ de altura, sendo a sua ocorrência registrada em toda a região Sudeste e também no sul da Bahia e em Mato Grosso do $\mathrm{Sul}^{5}$. É conhecida por ipê-roxo, ipê-roxo-de-sete-folhas, piúva (no Pantanal), dentre outras denominações populares. Possui madeira resistente e durável, própria para obras externas, sendo também utilizada para fins paisagísticos em função de suas flores de coloração roxa $^{5}$. A literatura registra a ocorrência de apenas dois trabalhos realizados com espécimens identificados como sendo T. heptaphylla: das folhas de um exemplar coletado no Rio Grande do Sul foi isolado um iridóide e do caule de um espécimen adquirido no Paraguai foram obtidos naftoquinonas, lignanas, prenil-naftalenos e outros compostos aromáticos ${ }^{3,6}$. O presente trabalho faz parte de um programa de levantamento e investigação da composição química de espécies que ocorrem no Cerrado e no Pantanal Sul-matogrossenses, tendo sido efetuado o estudo químico das cascas do caule de um exemplar de T. heptaphylla coletado no Pantanal. Neste descreve-se o isolamento de catorze substâncias, compreendendo dois triterpenos (1 e 2), cinco iridóides (3-7), um dialdeído ciclopentênico (8), um glicosídeo feniletanóide (9) e três derivados do ácido benzóico (1012), além de sitostenona (13) e sitosterol (14). O triterpeno 1 e o iridóide 7 são inéditos, o glicosídeo fenil etanóide $\mathbf{9}$ está sendo relatado pela primeira vez no gênero Tabebuia e as demais substâncias,

*e-mail: frgarcez@nin.ufms.br com exceção de 14, pela primeira vez na espécie.

A atividade antioxidante dos compostos isolados foi também avaliada neste trabalho.

\section{PARTE EXPERIMENTAL}

\section{Instrumentação e procedimentos gerais}

Para cromatografia em camada delgada analítica (CCD), utilizou-se sílica gel $60 \mathrm{G}(5-40 \mu \mathrm{m})$, em camadas de $0,25 \mathrm{~mm}$ de espessura, empregando-se como revelador solução de sulfato de cério IV em ácido sulfúrico concentrado. As separações cromatográficas em coluna foram realizadas utilizando-se gel de sílica 60 70-230 e 230-400 mesh, Sephadex LH-20 e XAD-2 (300-1200 $\mu \mathrm{m})$. As separações por cromatografia líquida de alta eficiência (CLAE) em condições semi-preparativas foram realizadas em coluna de sílica RP-18 Shim-Pack PREP-ODS(H) $(25$ x 250 mm, $5 \mu \mathrm{m})$, utilizando-se bomba ternária Shimadzu LC-6AD, com detector UV/VIS Shimadzu SPDV-6AV e monitorando-se a $254 \mathrm{~nm}$. Os espectros de RMN ${ }^{1} \mathrm{H}$ e ${ }^{13} \mathrm{C}$ (uni- e bidimensionais) foram obtidos em espectrômetro Bruker DPX-300 (300/75 MHz), utilizando-se $\mathrm{CDCl}_{3}$, $\mathrm{CD}_{3} \mathrm{OD}$ ou piridina- $d_{5}$ como solventes e TMS como padrão interno. Os espectros IV foram registrados em espectrômetro BomemHartmann \& Braun FT, tendo sido as amostras preparadas sob a forma de pastilhas de $\mathrm{KBr}$. As medidas de rotação óptica foram determinadas em polarímetro Perkin-Elmer 341.

\section{Material vegetal}

As cascas do caule de Tabebuia heptaphylla (Vell.) Toledo foram coletadas na Base de Estudos do Pantanal da Universidade Federal de Mato Grosso do Sul, localizada na região do Passo do Lontra, MS, em fevereiro de 2003. A identificação foi realizada por MSc. U. M. Resende (DBI/UFMS) e Dra. R. Farias-Singer (Instituto de Biologia/UNICAMP) e as exsicatas encontram-se depositadas no Herbário CG/MS da UFMS, sob o número 118999. 


\section{Extração e isolamento dos constituintes}

As cascas do caule secas e moídas de T. heptaphylla $(1,4 \mathrm{~kg})$ foram extraídas à temperatura ambiente com etanol. $\mathrm{O}$ extrato etanólico foi concentrado sob pressão reduzida até consistência xaroposa e em seguida, particionado entre hexano- $\mathrm{CH}_{3} \mathrm{CN}-\mathrm{CHCl}_{3}$ $\mathrm{H}_{2} \mathrm{O}$ 20:34:10:10, sendo obtidas 3 fases: hexânica (superior), $\mathrm{CH}_{3} \mathrm{CN}-\mathrm{CHCl}_{3}$ (intermediária) e aquosa (inferior).

A fase hexânica $(3,5 \mathrm{~g})$ foi submetida a uma coluna de gel de sílica 70-230 mesh, eluída com hexano e AcOEt em gradiente de polaridade crescente. Deste processo resultaram, após análise por CCD, 24 frações (H 1 $\rightarrow 24)$. A fração H1 forneceu 2 (164,8 mg), a fração H10 $(112,0 \mathrm{mg})$ continha predominantemente $\mathbf{1 3}$ e as frações H11 (27,9 mg) e H22 (38,8 mg) originaram, respectivamente, após cromatografia em coluna de Sephadex LH-20 eluída com $\mathrm{CHCl}_{3}-\mathrm{MeOH} 3: 2, \mathbf{1 4}(5,0 \mathrm{mg})$ e $\mathbf{1 2}(4,0 \mathrm{mg})$.

A fase $\mathrm{CH}_{3} \mathrm{CN}-\mathrm{CHCl}_{3}(15,1 \mathrm{~g})$ foi fracionada em coluna de gel de sílica 70-230 mesh, eluída com misturas de hexano-AcOEt e AcOEt$\mathrm{MeOH}$ em ordem crescente de polaridade, fornecendo, após análise por CCD, 21 frações (C $1 \rightarrow 21)$. Das frações C6 (483,0 mg) e C11 (406,2 mg) foram obtidos, respectivamente, após cromatografia em coluna de Sephadex LH-20 eluída com $\mathrm{CHCl}_{3}-\mathrm{MeOH}$ 3:2, 13 (86,4 $\mathrm{mg})$ e $11(17,0 \mathrm{mg})$. A fração C12 (716,3 mg) originou $\mathbf{1}(9,9 \mathrm{mg})$, após cromatografia em coluna de Sephadex LH-20 $\left(\mathrm{CHCl}_{3}-\mathrm{MeOH}\right.$ 3:2), seguida de cromatografia em coluna de gel de sílica 230-400 mesh, eluída com gradiente de $\mathrm{CHCl}_{3}$ e acetona. A fração C13 (3,3 g) foi recromatografada sucessivamente em coluna de Sephadex LH-20 eluída com $\mathrm{CHCl}_{3}-\mathrm{MeOH}$ 3:2, originando $\mathbf{1 0}(43,5 \mathrm{mg})$ e 12 (42,0 mg). Da fração C18 (804,6 mg) obteve-se 5 (151,8 mg), após cromatografia em coluna de Sephadex $\mathrm{LH}-20$ eluída com $\mathrm{CHCl}_{3}$ $\mathrm{MeOH} \mathrm{3:2.}$

A fase aquosa foi concentrada sob pressão reduzida e o resíduo obtido (170,2 g) foi extraído sucessivamente com AcOEt e $n$-BuOH, originando os extratos AcOEt e $n$-butanólico, respectivamente. $\mathrm{O}$ extrato AcOEt (145,9 g) foi cromatografado em uma coluna de XAD2, eluída com $\mathrm{H}_{2} \mathrm{O} \rightarrow \mathrm{MeOH} \rightarrow$ hexano $\rightarrow$ acetona, fornecendo, após análise por $\mathrm{CCD}, 12$ frações $(\mathrm{A} 1 \rightarrow 12)$. A fração A4 $(1,7 \mathrm{~g})$ foi submetida à cromatografia em coluna de Sephadex LH-20 eluída com $\mathrm{MeOH}$, fornecendo 7 (3,4 mg), após CLAE semi-preparativa (MeOH$\mathrm{H}_{2} \mathrm{O}$ 65:35) e 8 (9,7 mg), após cromatografia em coluna de gel de sílica 230-400 mesh, eluída com $\mathrm{CHCl}_{3}-\mathrm{MeOH} 5 \%$. A fração A5 (231,9 $\mathrm{mg}$ ) foi cromatografada em coluna de Sephadex LH-20 (MeOH), seguida de cromatografia em coluna de gel de sílica 230-400 mesh $\left(\mathrm{CHCl}_{3}-\mathrm{MeOH} 2 \%\right)$, resultando no isolamento de 5 (13,0 mg) e de $\mathbf{6}$ (7,7 mg), este após CLAE semi-preparativa (MeOH-H2O 70:30). O extrato $n$-butanólico $(1,73 \mathrm{~g})$ foi cromatografado em coluna de gel de sílica 70-230 mesh, utilizando-se como eluente hexano, seguido de AcOEt e depois, $\mathrm{MeOH}$, originando 5 frações $(\mathrm{B} 1 \rightarrow 5)$. Da fração B2 (383,7 mg) foi obtido 3 (9,9 mg), após cromatografia em coluna de Sephadex LH-20 (MeOH), seguida de cromatografia em coluna de gel de sílica 230-400 mesh $\left(\mathrm{CHCl}_{3}-\mathrm{MeOH} 8 \%\right)$, enquanto que a fração B4 (392,1 mg) forneceu 4 (14,1 mg) e 9 (9,9 mg), após sucessivos fracionamentos em coluna de Sephadex LH-20 (MeOH).

\section{$3 \beta, 6 \beta, 21 \beta$-triidroxiolean-12-eno (1)}

Sólido amorfo. $[\alpha]_{\mathrm{D}}^{23}:+17,4^{\circ}\left(\mathrm{CHCl}_{3} ; c 0,0019\right), \mathrm{IV}(\mathrm{KBr}) v_{\max }$ $\mathrm{cm}^{-1}: 3424,2927,1472,1190,758 . \mathrm{RMN}{ }^{1} \mathrm{H}$ e ${ }^{13} \mathrm{C}$ : Tabela 1.

\section{Ensaio de atividade antioxidante}

O teste foi realizado segundo metodologia descrita na literatura, empregando-se uma solução $0,47 \times 10^{-3} \mathrm{M}$ de $\alpha$-tocoferol como referência ${ }^{7}$. As amostras foram aplicadas em concentrações mola- res semelhantes em placas cromatográficas de sílica gel $\mathrm{G}$ e estas foram borrifadas com uma solução $0,02 \%$ de $\beta$-caroteno em $\mathrm{CH}_{2} \mathrm{Cl}_{2}$, seguidas por exposição à luz natural até descoloração do fundo.

Tabela 1. Dados de RMN ${ }^{1} \mathrm{H}(300 \mathrm{MHz})$ e ${ }^{13} \mathrm{C}(75 \mathrm{MHz})$ de 1 (piridina- $d_{5}$ )

\begin{tabular}{|c|c|c|c|c|}
\hline $\mathrm{C} / \mathrm{H}$ & $\delta_{\mathrm{H}}$ & $\delta_{\mathrm{C}}$ & $\begin{array}{c}\mathrm{HMBC} \\
{ }^{2} J_{\mathrm{CH}}\end{array}$ & ${ }^{3} J_{\mathrm{CH}}$ \\
\hline 1 & $1,60^{\mathrm{a}, \mathrm{b}}$ & 41,3 & & $\mathrm{H}-25$ \\
\hline 2 & $\begin{array}{l}1,85 \mathrm{~m}, \mathrm{H}-2_{\mathrm{ax}} \\
2,10 \mathrm{~m}\left(\mathrm{H}-2_{\mathrm{eo}}\right)\end{array}$ & 28,2 & & \\
\hline 3 & 3,46 dd $(11,8 ; 4,2)$ & 78,5 & $\mathrm{H}-2$ & H-23, H-24 \\
\hline 4 & - & 40,4 & H-23, H-24 & \\
\hline 5 & $0,95^{\mathrm{a}, \mathrm{c}}$ & 56,3 & & $\begin{array}{c}\mathrm{H}-23, \mathrm{H}-24, \\
\mathrm{H}-25\end{array}$ \\
\hline 6 & $4,86 \mathrm{sl}$ & 67,4 & & \\
\hline 7 & $1,70-1,80 \mathrm{~m}$ & 41,0 & & $\mathrm{H}-26$ \\
\hline 8 & - & 39,4 & $\begin{array}{c}\text { H-7a, H-7b, } \\
\text { H-26 }\end{array}$ & $\mathrm{H}-27$ \\
\hline 9 & $1,70-1,80 \mathrm{~m}$ & 48,5 & & H-25, H-26 \\
\hline 10 & - & 36,9 & $\mathrm{H}-9, \mathrm{H}-25$ & H-2 \\
\hline 11 & $2,05-2,10 \mathrm{~m}$ & 23,9 & H-9 & \\
\hline 12 & 5,37 tl $(3,6)$ & 123,1 & & \\
\hline 13 & - & 143,6 & & \\
\hline 14 & - & 42,4 & $\mathrm{H}-27$ & $\mathrm{H}-26$ \\
\hline 15 & $1,00 \mathrm{~m}$ & 26,4 & & $\mathrm{H}-27$ \\
\hline 16 & $1,25-1,35 \mathrm{~m}$ & 29,9 & & \\
\hline 17 & - & 35,0 & H-22, H-28 & \\
\hline 18 & $2,10-2,20 \mathrm{~m}$ & 47,1 & & H-22, H-28 \\
\hline 19 & $1,35^{a, d}$ & 47,6 & & H-29, H-30 \\
\hline 20 & - & 36,9 & $\begin{array}{c}\text { H-22, H-29, } \\
\text { H-30 }\end{array}$ & \\
\hline 21 & $3,84 \mathrm{tl}(8,0)$ & 72,8 & H-22 & H-29, H-30 \\
\hline 22 & $1,70-1,80 \mathrm{~m}$ & 46,5 & & H-28 \\
\hline 23 & $1,42 \mathrm{~s}$ & 28,5 & & H-3, H-24 \\
\hline 24 & $1,73 \mathrm{~s}$ & 18,0 & & H-3, H-23 \\
\hline 25 & $1,64 \mathrm{~s}$ & 17,2 & & $\mathrm{H}-9$ \\
\hline 26 & $1,54 \mathrm{~s}$ & 18,3 & & \\
\hline 27 & $1,22 \mathrm{~s}$ & 26,1 & & \\
\hline 28 & $0,94 \mathrm{~s}$ & 28,5 & & \\
\hline 29 & $1,22 \mathrm{~s}$ & 29,9 & & H-21, H-30 \\
\hline 30 & $1,14 \mathrm{~s}$ & 17,6 & & H-21, H-29 \\
\hline OH-6 & $5,51 \mathrm{dl}(3,6)$ & - & & \\
\hline
\end{tabular}

${ }^{a}$ valor aproximado de deslocamento químico obtido a partir dos espectros HMQC e HMBC; bencoberto pelo sinal da água; 'encoberto pelo sinal de $3 \mathrm{H}-28$; dencoberto pelo sinal de $3 \mathrm{H}-25$.

\section{RESULTADOS E DISCUSSÃO}

Através de partições do extrato etanólico das cascas do caule de Tabebuia heptaphylla, seguidas de fracionamentos cromatográficos, foram isolados dois triterpenos (1 e 2), cinco iridóides (3-7), um dialdeído ciclopentênico (8), um glicosídeo feniletanóide (9) e três derivados do ácido benzóico (10-12), além de dois esteróides (13 e 14) (Figura 1).

A substância $\mathbf{1}$ apresentou-se na forma de um sólido amorfo e seu espectro de RMN ${ }^{1} \mathrm{H}$ (Tabela 1) mostrou oito singletos na região entre $\delta$ 0,94-1,73 atribuíveis a grupos metila, um tripleto largo proporcional a um hidrogênio em $\delta 5,37(\mathrm{~J} 3,6 \mathrm{~Hz})$, sugestivo de hidrogênio olefínico, além de sinais referentes a três hidrogênios cada a $\delta 3,46(\mathrm{dd}, J 11,8$ e 4,2 Hz), 3,84 (tl, $J$ 8,0 Hz) e 4,86 (sl) atribuíveis a hidrogênios carbinólicos. O espectro de $\mathrm{RMN}{ }^{13} \mathrm{C}$ (Tabela 1) mostrou a presença de vinte e oito sinais (sendo dois deles correspondentes a dois carbo- 
nos cada) os quais, com o auxílio das informações fornecidas pelo espectro DEPT $135^{\circ}$, foram atribuídos a sete carbonos quaternários (sendo um deles olefínico), sete metínicos (sendo três oxigenados e um olefínico), oito metilênicos e oito metílicos. Estas informações indicaram que 1 se tratava de um triterpeno pentacíclico, sendo que os valores de deslocamento químico observados para os carbonos da ligação dupla trissubstituída ( $\delta 123,1$ e 143,6) definiram o esqueleto do tipo olean-12-eno ${ }^{8}$. Os sinais a $\delta 3,46 ; 4,86$ e 3,84 apresentaram correlações no espectro HMQC com os sinais de carbonos metínicos carbinólicos a $\delta 78,5,67,4$ e 72,8, respectivamente. Estes dados, aliados a uma banda de absorção a $3424 \mathrm{~cm}^{-1}$ no espectro IV, sugeriram a fórmula molecular $\mathrm{C}_{30} \mathrm{H}_{50} \mathrm{O}_{3}$ para $\mathbf{1}$. Na comparação dos dados de RMN ${ }^{1} \mathrm{H}$ e de ${ }^{13} \mathrm{C}$ de $1 \mathrm{com}$ os de triterpenos relacionados contendo os mesmos tipos de substituintes, verificou-se que $\mathbf{1}$ possui o mesmo padrão de substituição nos anéis A e B que o sumaresinolato de metila e o anel E idêntico ao do kuzusapogenol $\mathrm{C}$, indicando a localização dos grupos hidroxila em C-3, C-6 e C-2 $1^{9}$. A orientação equatorial do grupo hidroxila em C-3 foi indicada pela multiplicidade do sinal relativo ao hidrogênio carbinólico H-3 ( $\delta 3,46$, dd , $J 11,8$ e 4,2 Hz), enquanto que o singleto largo a $\delta 4,86$ relativo a H-6 revelou sua orientação equatorial e, conseqüientemente, a localização axial do grupo hidroxila em C-6. Da mesma forma, o tripleto largo a $\delta 3,84(1 \mathrm{H}, J 8,0 \mathrm{~Hz})$ indicou a localização do terceiro grupo hidroxila em C-21, apresentando orientação equatorial, o que pôde ser confirmado pela desproteção de C-20 e de C-22, assim como a proteção das metilas C-29 e C-30 (mais acentuada sobre C-30), quando os valores de deslocamento químico destes carbonos em 1 foram comparados com os de triterpenos sem substituintes no anel E,<smiles>CC(C)=CCCC(C)=CCCC1=CCCCC=C1CCCC(C)C</smiles><smiles>[R]c1ccc(C(=O)O[C@@H]2C[C@@](C)(O)[C@@H]3[C@@H]2C=CO[C@H]3OC)cc1[R]</smiles><smiles>[R]c1ccc(C(=O)O[C@@H]2C[C@@](C)(O)[C@H]3[C@H](OC)O[C@H](O)C[C@H]32)cc1[Y]</smiles>

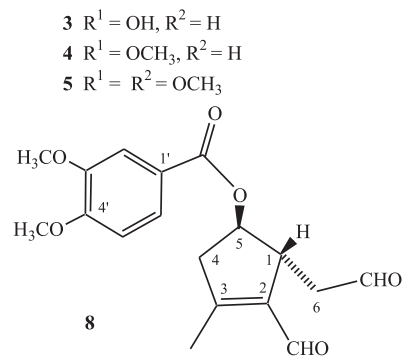

$6 \mathrm{R}^{1}=\mathrm{OH}, \mathrm{R}^{2}=\mathrm{H}$

$7 \mathrm{R}^{1}=\mathrm{R}^{2}=\mathrm{OCH}_{3}$

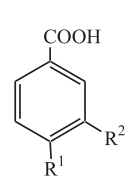

$10 \mathrm{R}^{1}=\mathrm{OH}, \mathrm{R}^{2}=\mathrm{H}$ $11 \mathrm{R}^{1}=\mathrm{OCH}_{3}, \mathrm{R}^{2}=\mathrm{H}$ $12 \mathrm{R}^{1}=\mathrm{R}^{2}=\mathrm{OCH}_{3}$

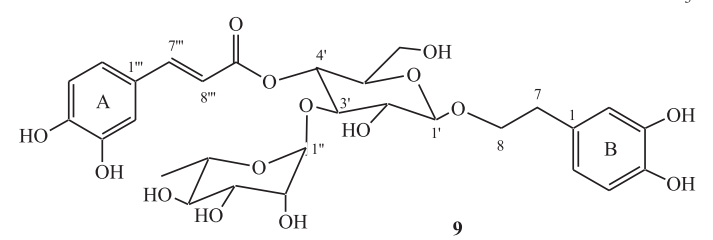

Figura 1. como por exemplo, $\beta$-amirina. As correlações à longa distância observadas no espectro HMBC para os hidrogênios carbinólicos e outros hidrogênios presentes em $\mathbf{1}$ (Tabela 1) corroboraram tais informações. Dessa forma, foi proposta para 1 a estrutura correspondente a $3 \beta, 6 \beta, 21 \beta$ triidroxiolean-12-eno, até então não relatada na literatura. Deve-se ressaltar também que, embora várias espécies de Tabebuia tenham sido quimicamente investigadas, raros são os registros de ocorrência de triterpenos nestas espécies, como por exemplo, em T. rosea, $T$ pentaphylla e T. aurea ${ }^{10,11}$.

Os dados espectrais de $\mathrm{RMN}{ }^{1} \mathrm{H}$ e ${ }^{13} \mathrm{C}$ das substâncias 3 a 5 apresentaram uma boa correlação com os dos iridóides 6- $O$ - $p$ hidroxibenzoilajugol, 6-O-p-metoxibenzoilajugol e 6-O-3", 4"dimetoxibenzoilajugol, respectivamente, já obtidos de Tabebuia avellanedae $^{12}$. Da mesma forma, as substâncias $\mathbf{1 0}$ a $\mathbf{1 2}$ foram identificadas como derivados do ácido benzóico, respectivamente, ácidos p-hidroxibenzóico, p-metoxibenzóico e 3,4-dimetoxibenzóico, anteriormente relatados em T. impetiginosa e/ou T. avellanedae $e^{4,13,14}$.

Os espectros de RMN ${ }^{1} \mathrm{H}$ e de ${ }^{13} \mathrm{C}$ de 6 (Tabela 2) mostraram-se semelhantes aos do iridóide $\mathbf{3}$, particularmente com relação ao anel de cinco membros e ao grupo para-hidroxibenzoíla, porém não apresentaram os sinais correspondentes ao resíduo de $\beta$-D-glucose em C-1 e à ligação dupla entre C-3 e C-4. Foi observada, no entanto, no espectro de $\mathrm{RMN}{ }^{1} \mathrm{H}$ de $\mathbf{6}$ a presença de dois grupos metoxila alifáticos $(\delta 3,44$ e 3,47$)$, um dubleto a $\delta 4,69(1 \mathrm{H}, J 5,6 \mathrm{~Hz})$, um duplo dubleto a $\delta 4,77$ $\left(1 \mathrm{H}, J 6,2\right.$ e 3,9 Hz), enquanto no espectro de RMN ${ }^{13} \mathrm{C}$, em associação com os dados fornecidos pelo espectro DEPT $135^{\circ}$, foram observados sinais de dois carbonos metínicos a $\delta 97,7$ e 97,8 e um metilênico a $\delta$ 29,5. Estas informações foram sugestivas da presença de duas funções acetálicas na estrutura de $\mathbf{6}$. Uma vez que as principais diferenças nos esqueletos dos iridóides $\mathbf{3}$ e $\mathbf{6}$ residiam no padrão de substituição do anel heterocíclico, foi possível propor a localização destas funções nas posições 1 e 3 . Assim, o dubleto a $\delta 4,69$ e o duplo dubleto a $\delta 4,77$ foram atribuídos a H-1 e a H-3, respectivamente $\left(\delta_{C} 97,7\right.$ e 97,8), e os hidrogênios em C-4 $\left(\delta_{C} 29,5\right)$ caracterizados como os multipletos nas regiões de $\delta 1,46-1,58$ e $\delta 1,94-2,06$. Assim, a substância 6 foi caracte-

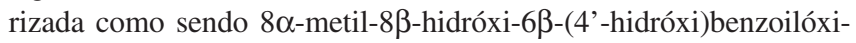
$1 \alpha, 3 \alpha$-dimetóxi-octaidro-ciclopenta[c]pirano, cujos dados espectrais, assim como o valor de rotação óptica $\left([\alpha]_{D}^{23}:-44,0^{\circ}\right)$ foram compatíveis com os citados na literatura para o mesmo iridóide isolado de Tabebuia avellanedae ${ }^{15}$. No entanto, deve ser revisado o valor de deslocamento químico atribuído a C-8 para o iridóide descrito naquele trabalho $(\delta 49,0)$, incoerente para um carbono oxigenado tetrassubstituído. A possibilidade de $\mathbf{6}$ ser um artefato produzido durante o processo de isolamento não pode ser excluída, embora tenha sido demonstrado que o iridóide obtido de $T$. avellanedae se tratava de um constituinte genuíno daquela planta ${ }^{15}$.

Os sinais do espectro de RMN ${ }^{1} \mathrm{H} \mathrm{e}{ }^{13} \mathrm{C}$ de 7 mostraram-se bastante semelhantes aos de $\mathbf{6}$, com exceção dos sinais relativos ao resíduo benzoíla em C-6, os quais foram substituídos pelos do grupo 3,4-dimetoxibenzoíla, já evidenciados no iridóide 5 (Tabela 2). Com base nestas informações e nas correlações presentes no espectro ${ }^{1} \mathrm{H}-{ }^{1} \mathrm{H}$ COSY entre os hidrogênios do anel heterocíclico, chegou-se à conlusão que 7 tratava-se do iridóide $8 \alpha$-metil- $8 \beta$ hidróxi-6 $\beta$-(3',4'-dimetóxi)benzoilóxi- $1 \alpha, 3 \alpha$-dimetóxi-octaidrociclopenta[c]pirano, ainda não relatado na literatura. Assim como em 6, a junção cis dos anéis A e B bem como as orientações $\beta$ de $\mathrm{H}-5$ e H-9 e $\alpha$ dos grupos metoxila em $\mathrm{C}-1$ e C-3 foram corroboradas pelas correlações evidenciadas no espectro NOESY de 7 entre H-5/H-9, H-1/H-9, H-3/H-5, H-3/H-4ß e H-1/H-4ß. Da mesma forma que 6, deve-se considerar a possibilidade do iridóide 7 ser um artefato resultante do hemiacetal correspondente. Iridóides que possuem funções acetálicas concomitantemente em C-1 e C-3, embora já tendo sido relatados em outras espécies de Bignoniaceae, 
Tabela 2. Dados de $\mathrm{RMN}{ }^{1} \mathrm{H}(300 \mathrm{MHz})$ e ${ }^{13} \mathrm{C}(75 \mathrm{MHz})$ de 6,7 e $8\left(\mathrm{CDCl}_{3}\right)$

\begin{tabular}{|c|c|c|c|c|c|c|}
\hline $\mathrm{C} / \mathrm{H}$ & $\begin{array}{c}\mathbf{6} \\
\delta_{\mathrm{H}}\end{array}$ & $\delta_{\mathrm{C}}$ & $\begin{array}{c}7 \\
\delta_{\mathrm{H}} \\
\end{array}$ & $\delta_{\mathrm{C}}$ & $\begin{array}{c}\mathbf{8} \\
\delta_{\mathrm{H}} \\
\end{array}$ & $\delta_{\mathrm{C}}$ \\
\hline 1 & $4,69 \mathrm{~d}(5,6)$ & $97,7^{\mathrm{a}}$ & $4,69 \mathrm{~d}(5,7)$ & $97,7^{\mathrm{a}}$ & $3,56 \mathrm{~m}$ & 45,9 \\
\hline 2 & - & - & - & - & - & 137,2 \\
\hline 3 & 4,77 dd $(6,2 ; 3,9)$ & $97,8^{\mathrm{a}}$ & 4,78 dd $(6,4 ; 3,9)$ & $97,9^{\mathrm{a}}$ & - & 161,0 \\
\hline 4 & $\begin{array}{l}1,46-1,58 \mathrm{~m} \mathrm{H}-4 \beta \\
1,94-2,06 \mathrm{~m} \mathrm{H}-4 \alpha\end{array}$ & 29,5 & $\begin{array}{c}1,55 \mathrm{~m} \mathrm{H}-4 \beta \\
1,98-2,04 \mathrm{~m} \mathrm{H}-4 \alpha\end{array}$ & 29,8 & $\begin{array}{c}3,24 \mathrm{dd}(19,7 ; 7,0) \mathrm{H}-4 \mathrm{a} \\
2,68 \mathrm{~m} \mathrm{H}-4 \mathrm{~b}\end{array}$ & 45,9 \\
\hline 5 & $2,74-2,86 \mathrm{~m}$ & 40,8 & $2,75-2,78 \mathrm{~m}$ & 40,7 & $5,15 \mathrm{dt}(7,0 ; 1,8)$ & 77,1 \\
\hline 6 & $5,21-5,23 \mathrm{~m}$ & 79,0 & $5,21-5,25 \mathrm{~m}$ & 79,2 & $\begin{array}{c}2,86 \text { ddd }(17,5 ; 4,2 ; 1,3) \text { H-6a } \\
2,68 \text { m H-6b }\end{array}$ & 45,0 \\
\hline 7 & $\begin{array}{l}2,33 \mathrm{dd}(15,3 ; 7,0) \mathrm{H}-7 \mathrm{a} \\
1,94-2,06 \mathrm{~m} \mathrm{H}-7 \mathrm{~b}\end{array}$ & 46,7 & $\begin{array}{c}2,34 \mathrm{dd}(15,1 ; 7,0) \mathrm{H}-7 \mathrm{a} \\
1,98-2,04 \mathrm{~m} \mathrm{H}-7 \mathrm{~b}\end{array}$ & 46,8 & - & - \\
\hline 8 & - & 79,7 & - & 79,2 & - & - \\
\hline 9 & 2,25 dd $(8,3 ; 5,6)$ & 52,5 & 2,25 dd $(8,3 ; 5,7)$ & 52,6 & - & - \\
\hline 10 & $1,41 \mathrm{~s}$ & 26,2 & $1,40 \mathrm{~s}$ & 26,2 & - & - \\
\hline $1^{\prime}$ & - & 122,1 & - & 122,7 & - & 122,5 \\
\hline $2^{\prime}$ & $7,83 \mathrm{~d}(8,6)$ & 131,8 & $7,49 \mathrm{~d}(2,0 \mathrm{~Hz})$ & 110,2 & $7,47 \mathrm{sl}$ & 111,7 \\
\hline $3^{\prime}$ & $6,78 \mathrm{~d}(8,6)$ & 115,2 & - & 150,0 & - & 148,7 \\
\hline 4 ' & - & 160,4 & - & 153,1 & - & 153,2 \\
\hline 5, & $6,78 \mathrm{~d}(8,6)$ & 115,2 & $6,85 \mathrm{~d}(8,5)$ & 112,0 & $6,86 \mathrm{~d}(8,5)$ & 110,2 \\
\hline $6^{\prime}$ & $7,83 \mathrm{~d}(8,6)$ & 131,8 & 7,63 dd $(8,5 ; 2,0)$ & 123,6 & $7,60 \mathrm{~d}(8,5)$ & 123,7 \\
\hline 7 ' & - & 166,1 & - & 166,0 & - & 166,1 \\
\hline $\mathrm{CH}_{3}-3$ & - & - & - & - & $2,21 \mathrm{~s}$ & 14,4 \\
\hline $\mathrm{CH}_{2} \mathrm{CHO}$ & - & - & - & - & $9,78 \mathrm{sl}$ & 200,7 \\
\hline $\mathrm{CHO}$ & - & - & - & - & $9,98 \mathrm{sl}$ & 187,5 \\
\hline $\mathrm{OCH}_{3}-1$ & $3,47 \mathrm{~s}$ & 55,7 & $3,47 \mathrm{~s}$ & 55,7 & - & - \\
\hline $\mathrm{OCH}_{3}^{3}-3$ & $3,44 \mathrm{~s}$ & 55,6 & $3,45 \mathrm{~s}$ & 55,5 & - & - \\
\hline $\mathrm{OCH}_{3}^{3}-3, \mathrm{OCH}_{3}-4^{\prime}$ & - & - & $3,90 \mathrm{~s} ; 3,91 \mathrm{~s}$ & 56,0 & $3,91 \mathrm{~s}$ & 56,0 \\
\hline
\end{tabular}

${ }^{a}$ Valores na mesma coluna que podem estar trocados. Dados de $\mathrm{RMN}{ }^{13} \mathrm{C}$ de 6 relatados por Awale et al. ${ }^{15}\left(\mathrm{CD}_{3} \mathrm{OD}, 100 \mathrm{MHz}\right): \delta_{\mathrm{C}} 26,9$ (C-10); 30,7 (C-4); 41,9 (C-5); 47,7 (C-7); 49,0 (C-8); 52,9 (C-9); 53,3 (OCH -3$) ; 56,0$ (OCH -1$) ; 80,3$ (C-6); 98,9 (C-1); 99,3 (C-3); 116,1 (C-3', 5'); 122,6 (C-1'); 132,6 (C-2',6'), 163,5 (C-4'); 168,5 (C-7').

como por exemplo, em Eccremocarpus scaber, Catalpa speciosa e Tabebuia aurea ${ }^{11,16}$, não são comuns no reino vegetal.

$\mathrm{O}$ espectro de RMN ${ }^{1}$ de $\mathbf{8}$ apresentou dois singletos de hidrogênios aldeídicos a $\delta 9,78$ e 9,98, enquanto que os sinais relativos às carbonilas correspondentes foram observados a $\delta 200,8$ e 187,5, sendo este último indicativo de uma carbonila $\alpha, \beta$-insaturada, o que foi corroborado pelos sinais dos carbonos de uma ligação dupla tetrassubstituída a $\delta 137,2$ e 161,0 (Tabela 2). No espectro IV, as bandas relativas às carbonilas aldeídicas foram observadas a $1719 \mathrm{e}$ $1672 \mathrm{~cm}^{-1}$. Destacaram-se ainda nos espectros de RMN ${ }^{1} \mathrm{H}$ e ${ }^{13} \mathrm{C}$ de 8 um singleto a $\delta 2,21$, sugestivo de uma metila vinílica $\left(\delta_{\mathrm{C}} 14,4\right)$, um multipleto a $\delta 2,68$ e um duplo duplo dubleto a $\delta 2,86$ (J 17,5; 4,8 e $1,3 \mathrm{~Hz}$ ), atribuíveis a hidrogênios metilênicos vizinhos à carbonila aldeídica não conjugada, além de sinais referentes a um resíduo 3,4dimetoxibenzoilóxi, já observado no iridóide $\mathbf{5}$. O sinal a $\delta 77,1$ foi atribuído ao carbono metínico ligado a este grupo, cujo hidrogênio foi caracterizado como um duplo tripleto a $\delta 5,15$ ( $J$ 7,0 e 1,8 Hz). Além destes sinais foram também observados no espectro de RMN ${ }^{1} \mathrm{H}$ de $\mathbf{8}$ um multipleto proporcional a um hidrogênio a $\delta 3,56$ e um duplo dubleto a $\delta 3,25(J 19,7$ e $7,0 \mathrm{~Hz})$ e no espectro de $\mathrm{RMN}{ }^{13} \mathrm{C}$ sinais relativos a dois carbonos metilênicos a $\delta 45,0$ e 45,9 e a um metínico a $\delta 45,9$. Com base nos dados apresentados, foi possível propor a estrutura $\mathbf{8}$ para esta substância, a qual se mostrou compatível com as correlações presentes no espectro ${ }^{1} \mathrm{H}-{ }^{1} \mathrm{H}$ COSY. Os dados espectrais de $\mathbf{8}$ apresentaram uma boa correlação com os relatados na literatura para o raro dialdeído ciclopentênico 2-formil-5-(3',4'-dimetoxibenzoilóxi)-3-metil-2-ciclopenteno-1-acetaldeído, o qual havia sido isolado anteriormente apenas de Tabebuia impetiginosa ${ }^{14}$.

A análise dos dados de $\mathrm{RMN}{ }^{1} \mathrm{H}$ e ${ }^{13} \mathrm{C}$ de 9 (Tabela 3) revelou a presença de dois anéis aromáticos do tipo 1,3,4-trissubstituído, carac- terizados pelos conjuntos de sinais a $\delta 6,77(\mathrm{~d}, J 8,2 \mathrm{~Hz}), 6,95$ $(\mathrm{dd}, J 8,2$ e 1,9 Hz) e 7,05 (d, $J 1,9 \mathrm{~Hz})$ [Anel A] e $\delta 6,68(\mathrm{sl}), 6,$, $(\mathrm{d}, J 8,3 \mathrm{~Hz})$ e 6,55 (dd, $J 8,3$ e 1,5 Hz) [Anel B]. Através do experimento HMQC, estes hidrogênios apresentaram correlação com os carbonos representados pelos sinais a $\delta 116,2,123,2,115,2,117,1,116,3$ e 121,4 , respectivamente. Também puderam ser observados dois dubletos a $\delta 6,27$ e 7,59 ( $J 15,9 \mathrm{~Hz})$ atribuíveis a hidrogênios olefínicos $\alpha$ e $\beta$, respectivamente, de um grupo carbonílico $\alpha, \beta$ insaturado $\left(\delta_{\mathrm{C}}\right.$ $168,3)$, os quais correlacionaram no espectro HMQC com os carbonos a $\delta 114,7$ e 148,0 , respectivamente. Estes dados, associados às correlações presentes no espectro HMBC entre os hidrogênios e carbonos do anel aromático $\mathrm{A}$ e os do grupo carbonila $\alpha, \beta$ insaturado foram compatíveis com a presença de um resíduo de cafeoíla na estrutura de 9. No espectro de RMN ${ }^{1} \mathrm{H}$, um tripleto largo a $\delta 2,78(J$ 7,2 Hz) e um multipleto entre $\delta 3,68-3,72$, correlacionados no espectro HMQC com os sinais de carbonos metilênicos a $\delta 36,6$ e 72,3, respectivamente, aliados às correlações existentes no espectro HMBC entre estes grupos e os sinais relativos ao anel aromático $\mathrm{B}$, foram indicativos da presença de um grupo 3,4-diidroxifeniletóxi em 9. A análise dos espectros de $\mathrm{RMN}{ }^{1} \mathrm{H} \mathrm{e}{ }^{13} \mathrm{C}$ também revelou a existência de dois resíduos de açúcar na estrutura, representados pelo sinal duplo a $\delta 4,37(J$ 7,5 Hz) e pelo singleto largo a $\delta 5,17$, os quais apresentaram correlações no espectro HMQC com os sinais a $\delta 104,2$ e 103,0. Esta informação, aliada aos valores de deslocamento químico e multiplicidades dos demais sinais relativos aos hidrogênios e carbonos dos dois açúcares, às correlações observadas a partir dos experimentos HMQC e HMBC e às informações da literatura ${ }^{17}$ permitiram identificar os dois resíduos de açúcar como sendo de $\beta$-D-glucose e $\alpha$-L-ramnose. Através das correlações presentes no espectro $\mathrm{HMBC}$ associadas a dados de modelos da literatura ${ }^{17}$, foram definidas as ligações dos grupos 3,4- 
Tabela 3. Dados de $\mathrm{RMN}{ }^{1} \mathrm{H}(300 \mathrm{MHz})$ e ${ }^{13} \mathrm{C}(75 \mathrm{MHz})$ de 9 $\left(\mathrm{CD}_{3} \mathrm{OD}\right)$

\begin{tabular}{|c|c|c|}
\hline $\mathrm{C} / \mathrm{H}$ & $\delta_{\mathrm{H}}$ & $\delta_{\mathrm{C}}$ \\
\hline 1 & - & 131,5 \\
\hline 2 & $6,68 \mathrm{sl}$ & 117,1 \\
\hline 3 & - & $146,1^{\mathrm{a}}$ \\
\hline 4 & - & $144,7^{\mathrm{a}}$ \\
\hline 5 & $6,67 \mathrm{~d}(8,1)$ & 116,3 \\
\hline 6 & 6,55 dd $(8,1 ; 1,7)$ & 121,3 \\
\hline 7 & 2,78 tl $(7,2)$ & 36,6 \\
\hline 8 & $3,70 \mathrm{~m}$ & 72,3 \\
\hline $1, "$ & - & 127,7 \\
\hline $2^{\prime}$, & $7,05 \mathrm{~d}(1,9)$ & 115,2 \\
\hline $3^{\prime \prime \prime}$ & - & 146,8 \\
\hline $4^{\prime \prime \prime}$ & - & 149,8 \\
\hline $5, "$ & $6,77 \mathrm{~d}(8,2)$ & 116,5 \\
\hline $6 "$, & 6,95 dd $(8,2 ; 1,9)$ & 123,2 \\
\hline $7^{\prime \prime \prime}$ & $7,59 \mathrm{~d}(15,9)$ & 148,0 \\
\hline $8^{\prime \prime}$ & $6,27 \mathrm{~d}(15,9)$ & 114,7 \\
\hline $9, "$ & - & 168,3 \\
\hline \multicolumn{3}{|c|}{ Resíduo de $\beta$-D-glucose } \\
\hline $1^{\prime}$ & $4,37 \mathrm{~d}(7,8)$ & 104,2 \\
\hline 2 ' & $3,39 \mathrm{~m}$ & 76,2 \\
\hline 3 , & 3,80 tl $(9,2)$ & 81,6 \\
\hline $4^{\prime}$ & $4,72 \mathrm{~m}$ & 70,6 \\
\hline 5 , & $3,55 \mathrm{~m}$ & 76,0 \\
\hline 6 ' & $3,55 \mathrm{~m}$ & 62,4 \\
\hline \multicolumn{3}{|c|}{ Resíduo de $\alpha$-L-ramnose } \\
\hline $1 "$ & $5,17 \mathrm{sl}$ & 103,0 \\
\hline $2 ”$ & $4,01 \mathrm{~m}$ & 72,3 \\
\hline $3 "$ & $3,90 \mathrm{~m}$ & 72,0 \\
\hline $4 "$ & $3,39 \mathrm{~m}$ & 73,8 \\
\hline $5 ”$ & $3,55 \mathrm{~m}$ & 70,4 \\
\hline $6 "$ & $1,08 \mathrm{~d}(6,1)$ & 18,5 \\
\hline
\end{tabular}

${ }^{a}$ valores que podem estar trocados

diidroxifeniletoxila e cafeoíla em C-1' e C-4' do resíduo de glucose, respectivamente, enquanto que a correlação entre o hidrogênio anomérico da ramnose e C-3' da glucose caracterizou a ligação do tipo 1" $\rightarrow 3$ ' entre os dois resíduos de açúcar. Assim, a estrutura de 9 foi definida como $\beta$ (3,4-diidroxifenil)-etil- $O$ - $\alpha$-L-ramnopiranosil-(1' $\rightarrow 3^{\prime}$ ')- $\beta$-D-(4'- $O$ cafeoil)-glucopiranosídeo, cujos dados espectrais mostraram-se compatíveis com os da substância denominada verbascosídeo, obtida de Orobanche rapum-genistae (Orobanchaceae) e relatada em espécies de Bignoniaceae, como por exemplo, Barnettia kerri e Markhamia stipulata ${ }^{18}$. Apesar de já ter sido descrita a ocorrência de glicosídeos feniletanóides em Tabebuia impetiginosa ${ }^{19}$, trata-se do primeiro relato do isolamento do verbascosídeo de uma espécie de Tabebuia.

Esqualeno (2), sitosterol (13) e sitostenona (14), de ocorrência comum em outras espécies vegetais, foram identificados com base nos respectivos dados espectrais de $\mathrm{RMN}{ }^{1} \mathrm{He} \mathrm{e}^{13} \mathrm{C}$, assim como por comparação com amostra autêntica e/ou com os relatados na literatura ${ }^{20}$.

Ao serem submetidos ao ensaio de atividade antioxidante, através da inibição da oxidação do $\beta$-caroteno em placas de $\mathrm{CCD}$, o iridóide 6 apresentou forte atividade, os iridóides 3 e 4 e o verbascosídeo (9) inibiram moderadamente a oxidação do $\beta$-caroteno, enquanto que o iridóide $\mathbf{5}$ apresentou fraca atividade antioxidante. Os demais compostos mostraram-se inativos neste ensaio.

\section{CONCLUSÕES}

O estudo químico das cascas do caule de um exemplar de Tabebuia heptaphylla coletado no Pantanal de Mato Grosso do Sul resultou no isolamento de catorze substâncias, compreendendo dois triterpenos, cinco iridóides, um dialdeído ciclopentênico, um glicosídeo feniletanóide, três derivados do ácido benzóico e dois esteróides. Nenhuma destas substâncias, com exceção do esteróide sitosterol, havia sido obtida em estudos anteriores realizados com dois espécimens identificados como T. heptaphylla e coletados em outras localidades. O triterpenóide $3 \beta, 6 \beta, 21 \beta$-triidroxiolean-12-eno

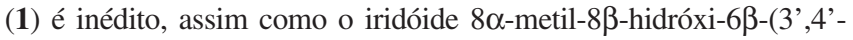
dimetóxi)benzoilóxi-1 $\alpha, 3 \alpha$-dimetóxi-octaidro-ciclopenta[c]pirano (7), sendo que existem poucos relatos sobre a ocorrência de triterpenos em espécies de Tabebuia. O glicosídeo feniletanóide verbascosídeo (9) está sendo descrito pela primeira vez no gênero Tabebuia, enquanto que o dialdeído ciclopentênico 2-formil-5-(3',4' dimetoxibenzoilóxi)-3-metil-2-ciclopenteno-1-acetaldeído (8) e o iridóide $8 \alpha$-metil-8 $\beta$-hidróxi-6 $\beta$-(4'-hidróxi)benzoilóxi-1 $\alpha, 3 \alpha$ dimetóxi-octaidro-ciclopenta[c]pirano (6) foram relatados somente em T. impetiginosa e T. avellanedae, respectivamente. Iridóides que possuem funções acetálicas concomitantes em C-1 e C-3, tais como em 6 e 7, embora encontrados em outras espécies de Bignoniaceae, não são comuns dentre os diversos já obtidos de plantas superiores.

\section{AGRADECIMENTOS}

À CAPES e PROPP-UFMS pelo apoio financeiro e bolsa de mestrado (T. S. Mahmoud) e à Dra. R. Farias-Singer (UNICAMP) pela identificação do material botânico.

\section{REFERÊNCIAS}

1. Gentry A. H.; Flora Neotropica. Monogr.25, The New York Botanical Garden: New York, 1980; Joly, A. B.; Introdução à Taxonomia Vegetal, Ed. Nacional: São Paulo, 1981.

2. Oliveira, A. B.; Raslan, D. S.; Miraglia, M. C. M.; Mesquita, A. A. L.; Zani, C. L.; Ferreira, D. T.; Maia, J. G. S.; Quim. Nova 1990, 13, 302.

3. von Poser, G. L.; Schripsema, J.; Henriques, A. T.; Jensen, S. R.; Biochem. Syst. Ecol. 2000, 28, 351.

4. Burnett, A. R.; Thomson, R. H.; J. Chem. Soc. C 1967, 2100.

5. Lorenzi, H.; Árvores Brasileiras: Manual de Identificação e Cultivo de Plantas Arbóreas Nativas do Brasil, $4^{\text {a }}$ ed., Instituto Plantarum: Nova Odessa, 2002, vol. 1

6. Schmeda-Hirschmann, G.; Papastergiou, F.; Z. Naturforsch., C: J. Biosci. 2003, 58, 495.

7. Pratt, D. E.; Miller, E. E.; J. Am. Oil Chem. Soc. 1984, 61, 1064.

8. Olea, R. S. G.; Roque, N. F.; Quim. Nova 1990, 13, 278.

9. Mahato, S. B.; Kundu, A. P.; Phytochemistry 1994, 37, 1517; Ohtani, P. K.; Ogawa, K.; Kasai, R.; Yang, C-R, K.; Zhou, J.; Tanaka, O.; Phytochemistry 1992, 31, 1747.

10. Oliveira, M. E. O.; Lemos, T. L. G.; Braz Filho, R.; Rev. Bras. Farm. 1999, 80, 46; Bishay, D. W.; Abdel-Baky, A. M.; Ross, S. A.; Ibrahim, Z. Z.; Bull. Pharm. Sci. 1987, 10, 1.

11. Guerbas Neto, P.; Dissertação de Mestrado, Universidade Federal de Mato Grosso do Sul, Brasil, 2002.

12. Nakano, K.; Maruyama, K.; Murakami, K.; Takaishi, Y.; Tomimatsu, T.; Phytochemistry 1993, 32, 371.

13. Kreher, B.; Lotter, H.; Cordell, G. A.; Wagner, H.; Planta Med. 1988, 54, 562; Burnett, A. R.; Thomson, R. H.; J. Chem. Soc. C 1967, 2110.

14. Koyama, J.; Morita, I.; Tagahara, K.; Hirai, K-I.; Phytochemistry 2000, 53, 869.

15. Awale, S.; Kawakami, T.; Tezuka, Y.; Ueda, J.; Tanaka, K.; Kadota, S.; Chem. Pharm. Bull. 2005, 53, 710.

16. Boros, C. A.; Stermitz, F. R.; J. Nat. Prod. 1990, 53, 1055.

17. Agrawal, P. K.; Phytochemistry 1992, 31, 3307.

18. Andary, C.; Wylde, R.; Laffite, C.; Privat, G.; Winternitz, F.; Phytochemistry 1982, 21, 1123; Kanchanapoom, T.; Kasai, R.; Yamasaki, K.; Phytochemistry 2002, 59, 565; Kanchanapoom, T.; Kasai, R.; Yamasaki, K.; Phytochemistry 2002, 59, 557.

19. Warashina, T.; Nagatani, Y.; Noro, T.; Phytochemistry 2004, 65, 2003.

20. Pouchert, C. J.; Behnke, J.; The Aldrich Library of ${ }^{13} \mathrm{C}$ and ${ }^{1} \mathrm{H}$ FTNMR Spectra, $1^{\text {st }}$ ed., Aldrich Chemical Company, Inc.: Milwaukee, 1993, vol. 1. 